\title{
Alteração da função renal em pacientes com Hipertensão Arterial Sistêmica: prevalência e fatores associados
}

\author{
Change in renal function of patients with Systemic Arterial Hypertension: prevalence and \\ associated factors
}

Cambio de la función renal en pacientes con Hipertensión Arterial Sistémica: prevalencia y factores asociados

Vitória Fernandes Rezende ${ }^{1 *}$, Victor Lucas Ferreira Correa ${ }^{1}$, Ana Carolina Solino Mourão1, Beatriz Malatesta Werneck ${ }^{1}$, Ioly Gondim dos Reis ${ }^{1}$, Maria Paula Schettine Catta Preta ${ }^{1}$, Víctor Jacometti ${ }^{1}$, Anna Marcella Neves Dias ${ }^{1}$, Nathália Barbosa do Espírito Santo Mendes ${ }^{1}$, Alice Mussi ${ }^{1}{ }^{2}$.

\section{RESUMO}

Objetivo: Identificar a alteração da Taxa de Filtração Glomerular estimada (TFGe) e os fatores associados em pacientes com Hipertensão Arterial Sistêmica. Métodos: Estudo transversal, retrospectivo, realizado através de prontuários de 280 pacientes hipertensos internados em um Hospital da região da Zona da Mata de Minas Gerais, entre janeiro de 2020 e março de 2021. Foi realizado o cálculo da TFGe através da equação desenvolvida pelo Chronic Kidney Disease Epidemiology Collaboration (CKD-EPI). Dividiu-se os pacientes em dois grupos, com e sem alteração da taxa de filtração glomerular sendo avaliados os fatores relacionados à cada um destes. Resultados: Do total de participantes, 27,86\% apresentaram TFGe alterada, sendo 0 padrão do paciente hipertenso com maior risco de desenvolver DRC caracterizado como: homem, branco, com 60 anos ou mais de idade. Conclusão: A prevalência de alteração da TFGe em hipertensos é alta, e tem como fator associado: Diabetes Mellitus, distúrbios hidroeletrolíticos e maior dificuldade em controlar a pressão arterial.

Palavras-chave: Hipertensão, Taxa de filtração glomerular, Doença renal.

\section{ABSTRACT}

Objective: To identify changes in estimated Glomerular Filtration Rate (eGFR) and associated factors in patients with Systemic Arterial Hypertension. Methods: Cross-sectional, retrospective study, performed through the medical records of 280 hypertensive patients admitted to a Hospital in the Zona da Mata region of Minas Gerais, between January 2020 and March 2021. The eGFR was calculated by the equation developed by the Chronic Kidney Disease Epidemiology Collaboration (CKD-EPI). The patients were divided into two groups, with changes and without changes in the glomerular filtration rate, and the factors related to each of these were evaluated. Results: Of the total number of participants, $27.86 \%$ had altered eGFR, with the pattern of hypertensive patients at higher risk of developing CKD characterized as: men, white, aged 60 years or over. Conclusion: The prevalence of eGFR alteration in hypertensive patients is high, and its associated factors are: DM, electrolyte disturbances and greater difficulty in controlling blood pressure.

Keywords: Hypertension, Glomerular filtration rate, Kidney disease.

${ }^{1}$ Centro Universitário Presidente Antônio Carlos (UNIPAC), Juiz de Fora - MG.

*E-mail: fr.vivi@hotmail.com

${ }^{2}$ Hospital Dr. João Felício, Juiz de Fora - MG.

SUBMETIDO EM: 12/2021

PUBLICADO EM: 12/2021 


\section{RESUMEN}

Objetivo: Identificar cambios en la Tasa de Filtración Glomerular estimada (TFGe) y factores asociados en pacientes con Hipertensión Arterial Sistémica. Métodos: Estudio transversal, retrospectivo, realizado a través de la historia clínica de 280 pacientes hipertensos hospitalizados en un Hospital de la Zona da Mata de Minas Gerais, entre enero de 2020 y marzo de 2021. La TFGe se calculó utilizando la ecuación desarrollada por la Colaboración en Epidemiología de la Enfermedad Renal Crónica (CKD-EPI). Los pacientes se dividieron en dos grupos, con y sin cambios en la tasa de filtrado glomerular, y se evaluaron los factores relacionados con cada uno de ellos. Resultados: Del total de participantes, el 27,86\% presentaba TFGe alterada, caracterizándose el patrón de los pacientes hipertensos con mayor riesgo de desarrollar ERC: hombre, raza blanca, 60 años o más. Conclusión: La prevalencia de alteración de la TFGe en pacientes hipertensos es alta y sus factores asociados son: DM, alteraciones electrolíticas y mayor dificultad en el control de la presión arterial.

Palabras clave: Hipertensión, Tasa de filtración glomerular, Enfermedad renal.

\section{INTRODUÇÃO}

A Hipertensão Arterial Sistêmica (HAS) é uma condição clínica multifatorial prevalente no Brasil e no mundo que se caracteriza pela elevação sustentada da Pressão Arterial (PA) acima ou igual a $140 \mathrm{mmHg}$ para sistólica e/ou $90 \mathrm{mmHg}$, para diastólica (BARROSO WKS, et al., 2020; FALCÃO AS, et al., 2018; BARROSO WKS, et al., 2020). Segundo dados da Organização Mundial da Saúde (OMS), mais de um bilhão de pessoas no mundo possuem HAS, sendo que a maioria desconhece sua condição (PAN AMERICAN HEALTH ORGANIZATION, 2020).

Apesar de sua etiologia imprecisa, vários são os fatores de risco associados a HAS, dentre eles pode-se destacar o sedentarismo, idade, tabagismo, ingestão excessiva de sódio, história familiar, raça, gênero, estresse, etilismo, excesso de peso, obesidade e baixo consumo de potássio (OLIVEIRA JL, et al., 2021; BARROSO WKS, et al., 2020). Fatores sociais e físicos também estão associados à HAS (CARVALHO MV, et al., 2013).

Por ser uma doença de evolução lenta e assintomática, com tratamento prolongado, esquemas terapêuticos complexos e presença de efeitos colaterais, há uma grande dificuldade de adesão ao tratamento da HAS e controle pressórico (FALCÃO AS, et al., 2018; GOUVEIA MMA, et al., 2018; BARRETO MS, et al., 2014). Cerca de um terço dos doentes não aderem ao tratamento, por fatores próprios ou relacionados a equipe multiprofissional de saúde e principalmente ao contexto socioeconômico e cultural que estão inseridos (OLIVEIRA BL, et al., 2020; FIGUEIREDO NN e ASSAKURA L, 2010).

Tais dados são de grande impacto para a prevenção do acometimento de lesões de órgão alvo, como a Doença Renal Crônica (DRC), condição prevalente que possui como principal etiologia no Brasil, a HAS (BARROSO WKS, et al., 2020; MARTINS NETO UR, et al., 2021). Por também ser fator de risco para a HAS, DRC e HAS possuem uma relação de mão dupla que envolve diversos mecanismos, sendo os principais: alteração no equilíbrio hidroeletrolítico, ativação do Sistema Renina-angiotensina-aldosterona e do Sistema Nervoso Autônomo Simpático, e disfunção endotelial (SANTOS TMP e VASCONCELOS SML, 2012; OLIVEIRA FJS, et al., 2019; SOUZA SR, et al., 2020; MORAES CE, et al., 2009; BUCHARLES SGE, et al., 2019).

Os rins possuem mecanismos autorreguladores que conseguem proteger seu funcionamento frente ao aumento sistêmico agudo dos níveis pressóricos arteriais, a exemplo da contração da arteríola glomerular aferente. Dessa forma, a transferência do aumento da pressão para a microvasculatura renal não acontece, constituindo-se um fator protetor dos glomérulos. Entretanto, se o aumento da pressão ocorre de forma mais severa, esse mecanismo torna-se insuficiente e, consequentemente, passa a permitir a transmissão da elevação da PA para os capilares glomerulares, causando injúria renal. Como resultado, as alterações vasculares como a diminuição do diâmetro do lúmen das arteríolas, colaboram com a manutenção elevada da PA, através do aumento da resistência vascular (MENNUNI S, et al., 2013). 
A detecção precoce de alterações da função renal é muito importante, já que pequenas elevações da creatinina sérica podem traduzir perda significativa da função renal, e o tratamento poderá estabilizar ou retardar a evolução da maioria das lesões renais. Neste aspecto, a determinação da Taxa de Filtração Glomerular estimada (TFGe) pelo clearance de creatinina, é mais precisa do que a simples dosagem da creatinina sérica (SARAFIDIS PA, et al., 2017).

Todavia, nem sempre uma TFGe normal traduz ausência de lesão renal, como por exemplo a Nefropatia Diabética em estágio primário, onde é marcante a hiperfiltração glomerular (REZENDE NETA DS, et al., 2012). É importante salientar também, que, se tratando de alteração da TFGe, não somente a HAS, mas fatores como, idade avançada, polifarmácia, complicações durante a internação hospitalar e uso de contraste para realização de exames, podem levar à sua alteração aumentando o risco de injúria renal (CERQUEIRA DP, et al., 2014; AOKI BB, et al., 2014). Além da TFGe, pode-se lançar mão da avaliação dos níveis séricos dos íons sódio e potássio, que nesses pacientes poderão estar aumentados devido à falha funcional dos rins, assim como da presença de proteinúria (SANTOS TMP e VASCONCELOS SML, 2012; PINHO NA, et al., 2015).

O objetivo do estudo foi identificar a prevalência de alteração da TFGe e os fatores associados em hipertensos.

\section{MÉTODOS}

Foi realizado um estudo observacional retrospectivo, transversal com dados de 280 pacientes no período de janeiro de 2020 a março de 2021, para identificar a prevalência de alteração da função renal em hipertensos internados em um Hospital da região da Zona da Mata de Minas Gerais.

Os dados dos pacientes foram analisados retrospectivamente através de prontuários eletrônicos, sendo incluídos na pesquisa aqueles com mais de 18 anos que estavam internados, previamente diagnosticados com HAS, desde que houvesse pelo menos um registro de PA e de exame laboratorial de creatinina sérica no prontuário. Foram excluídos da pesquisa os pacientes sem diagnóstico prévio de HAS e/ou com faixa etária menor que 18 anos e/ou ausência de creatinina sérica e/ou ausência da PA, sendo excluído um total de 27 prontuários.

A coleta de informações nos prontuários eletrônicos via sistema TASY incluiu: número de prontuário, nome completo, sexo, etnia, idade, PA, classes medicamentosas de anti-hipertensivos e fatores de risco para HAS como DM, dislipidemia e tabagismo. Além disso, foram obtidos os dados de exames laboratoriais de creatinina, sódio e potássio séricos. Sendo considerado dentro da normalidade os níveis séricos de 135 a 145 $\mathrm{mEq} / \mathrm{L}$ para sódio, de 3,5 a $5,5 \mathrm{mEq} / \mathrm{L}$ para potássio, enquanto o valor da creatinina foi utilizado para estimar a TFGE.

Para classes medicamentosas de anti-hipertensivos considerou-se: Diuréticos de alça e tiazídicos, Beta bloqueadores, Bloqueadores dos canais de cálcio, Inibidores da enzima conversora de angiotensina, Bloqueadores do receptor de angiotensina, Vasodilatador direto, Alfa-agonistas de ação central, Alfabloqueadores adrenérgicos. Foi avaliado também a quantidade de classes utilizadas por cada paciente.

Para PA em mmHg, foram considerados: PA normal quando Pressão Arterial Sistólica (PAS) $<120$ e Pressão Arterial Diastólica $(P A D)<80$, e para PA alterada, valores maiores ou iguais a 120 para PAS e 80 para PAD.

A TFGe foi estimada utilizando a equação desenvolvida pelo Chronic Kidney Disease Epidemiology Collaboration (CKD-EPI) sendo classificados com alterações da função renal presente aqueles com valores menores de $60 \mathrm{~mL} / \mathrm{min} / 1,73 \mathrm{~m}^{2}$ e ausente aqueles com valores maiores ou igual a $60 \mathrm{~mL} / \mathrm{min} / 1,73 \mathrm{~m}^{2}$.

Os dados foram armazenados no programa Excel 365, Microsoft Corporation®USA. Para a análise estatística, foi utilizado o programa SPSS 23.0, IBM®SPSS Statistics. Medidas de posição e tendência central foram utilizadas para a descrição de variáveis contínuas e proporções para as variáveis categóricas estudadas. 
$\mathrm{Na}$ análise com variáveis categóricas para verificar diferenças entre duas amostras independentes foi utilizado o teste de qui-quadrado de Pearson. Nas variáveis contínuas após verificar a normalidade através do teste de Shapiro Wilk, em aquelas com distribuição normal foram investigadas diferenças através do teste $T$ de igualdade de duas amostras independentes.

$\mathrm{Na}$ análise do p-valor e os intervalos de confiança o valor crítico foi definido em 95\%.

Este estudo foi aprovado pelo Comitê de Ética em Pesquisa com Seres Humanos do Centro Universitário Presidente Antônio Carlos, Barbacena - MG (Número do parecer: 4.157.071).

\section{RESULTADOS}

Ao analisar a presença de alteração da função renal em pacientes hipertensos internados e os fatores relacionados, observou-se que em um total de 280 pacientes, $202(72,14 \%)$ apresentaram TFGe $\geq 60$ $\mathrm{mL} / \mathrm{min} / 1,73 \mathrm{~m}^{2}$ e $78(27,86 \%)$ com TFGe $<60 \mathrm{~mL} / \mathrm{min} / 1,73 \mathrm{~m}^{2}$ (Tabela 1).

Com relação às variáveis sociodemográficas, houve prevalência de homens em ambos os grupos. Quanto à idade, ambos grupos eram compostos em sua maioria (67,5\%) por pacientes com mais de 60 anos, com predominância para os pacientes com alteração da TFGe $(83,3 \%)$. De acordo com a raça, foi verificado um predomínio da pele branca em ambos os grupos, $69,2 \%$ para aqueles com alteração da TFGe, e $62,4 \%$ para o outro grupo.

Quanto às comorbidades associadas, foi possível observar uma maior prevalência de DM no grupo com alteração da TFGe (44,9\%). Para a dislipidemia, não houve diferença entre os grupos. Dentro de hábitos e vícios, foi observado um maior percentual de tabagistas no grupo sem alteração da TFGe $(19,3 \%$ versus 15,4\%). Já no que diz respeito aos índices de sódio e potássio alterados, houve predomínio, 28,60\% e 22,90\% respectivamente, no grupo que possui alteração da TFGe.

Tabela 1 - Fatores associados a hipertensos com e sem alteração da taxa de filtração glomerular estimada.

\begin{tabular}{|c|c|c|c|c|c|c|}
\hline \multirow{3}{*}{ Variáveis } & \multicolumn{6}{|c|}{ TFGe } \\
\hline & \multicolumn{2}{|c|}{$\begin{array}{l}\text { Não alterada } \\
n=202\end{array}$} & \multicolumn{2}{|c|}{$\begin{array}{c}\text { Alterada } \\
n=78\end{array}$} & \multicolumn{2}{|c|}{$\begin{array}{l}\text { Todos } \\
n=280\end{array}$} \\
\hline & $\mathbf{n}$ & $\%$ & $\mathbf{n}$ & $\%$ & $\mathbf{n}$ & $\%$ \\
\hline \multicolumn{7}{|l|}{ Sexo } \\
\hline Masculino & 143 & $70,80 \%$ & 49 & $62,80 \%$ & 192 & $68,60 \%$ \\
\hline Feminino & 59 & $29,20 \%$ & 29 & $37,20 \%$ & 88 & $31,40 \%$ \\
\hline \multicolumn{7}{|l|}{ Idade } \\
\hline$<60$ anos & 78 & $38,60 \%$ & 13 & $16,70 \%$ & 91 & $32,50 \%$ \\
\hline$>=60$ anos & 124 & $61,40 \%$ & 65 & $83,30 \%$ & 189 & $67,50 \%$ \\
\hline [Média +/- DP] & $63,3+/-12,0$ & & $71,6+/-11,9$ & & $65,6+/-12,5$ & \\
\hline \multicolumn{7}{|l|}{ Cor de Pele } \\
\hline Branca & 126 & $62,40 \%$ & 54 & $69,20 \%$ & 180 & $64,30 \%$ \\
\hline Não branca & 76 & $37,60 \%$ & 24 & $30,80 \%$ & 100 & $35,70 \%$ \\
\hline Tabagistas & 39 & $19,30 \%$ & 12 & $15,40 \%$ & 51 & $18,20 \%$ \\
\hline Diabetes & 72 & $35,60 \%$ & 35 & $44,90 \%$ & 107 & $38,20 \%$ \\
\hline Dislipidemia & 98 & $48,50 \%$ & 37 & $48,10 \%$ & 135 & $48,40 \%$ \\
\hline Sódio Alterado & 12 & $10,30 \%$ & 10 & $28,60 \%$ & 22 & $14,50 \%$ \\
\hline Potássio Alterado & 13 & $11,10 \%$ & 8 & $22,90 \%$ & 21 & $13,80 \%$ \\
\hline
\end{tabular}

Fonte: Rezende VF, et al., 2021.

Os dados da Tabela 2 demonstraram que a maior parte dos pacientes, tanto sem alteração da TFGe, quanto com alteração da TFGe utilizam de dois a três medicamentos anti-hipertensivo, respectivamente $47 \%$ e $42,3 \%$. Cerca de um quarto dos pacientes de toda a amostra estava sem uso de qualquer medicação antihipertensiva. Entre os principais medicamentos anti-hipertensivos utilizados por pacientes sem alteração da TFGe, estão os bloqueadores do receptor AT1 da angiotensina ( $50 \%)$, seguido pelo beta bloqueador (32,2\%). Já entre os pacientes hipertensos com alteração da TFGe, a medicação de escolha também foi o bloqueador do receptor AT1 da angiotensina (42,3\%), seguido do beta bloqueador $(38,5 \%)$ e diuréticos de alça $(30,8 \%)$. 
Tabela 2 - Anti-hipertensivos usados por hipertensos com e sem alteração da taxa de filtração glomerular estimada.

\begin{tabular}{|c|c|c|c|c|c|c|c|}
\hline \multirow{3}{*}{ Variáveis } & \multicolumn{7}{|c|}{ TFGe } \\
\hline & \multicolumn{2}{|c|}{$\begin{array}{l}\text { Não alterada } \\
n=202\end{array}$} & \multicolumn{2}{|c|}{$\begin{array}{l}\text { Alterada } \\
\mathrm{n}=78\end{array}$} & \multicolumn{2}{|c|}{$\begin{array}{l}\text { Todos } \\
\mathrm{n}=280\end{array}$} & \multirow[t]{2}{*}{$p$-valor } \\
\hline & $\mathbf{n}$ & $\%$ & $\mathbf{n}$ & $\%$ & $\mathbf{n}$ & $\%$ & \\
\hline \multicolumn{8}{|l|}{ Número de anti-hipertensivos } \\
\hline Nenhum & 49 & $24,30 \%$ & 22 & $28,20 \%$ & 71 & $25,40 \%$ & 0,301 \\
\hline Um & 45 & $22,30 \%$ & 6 & $7,70 \%$ & 51 & $18,20 \%$ & 0,004 \\
\hline Dois a três & 95 & $47,00 \%$ & 33 & $42,30 \%$ & 128 & $45,70 \%$ & 0,283 \\
\hline Mais de três & 13 & $6,40 \%$ & 17 & $21,80 \%$ & 30 & $10,70 \%$ & 0 \\
\hline \multicolumn{8}{|l|}{ Classes } \\
\hline Diuréticos tiazídicos & 41 & $20,30 \%$ & 11 & $14,10 \%$ & 52 & $18,60 \%$ & 0,153 \\
\hline Diuréticos de alça & 33 & $16,30 \%$ & 24 & $30,80 \%$ & 57 & $20,40 \%$ & 0,006 \\
\hline Beta bloqueador & 65 & $32,20 \%$ & 30 & $38,50 \%$ & 95 & $33,90 \%$ & 0,196 \\
\hline $\begin{array}{l}\text { Bloqueadores dos canais de } \\
\text { cálcio }\end{array}$ & 23 & $11,40 \%$ & 12 & $15,40 \%$ & 35 & $12,50 \%$ & 0,24 \\
\hline $\begin{array}{l}\text { Bloqueador do receptor } \mathrm{AT}^{*}{ }^{*} \text { da } \\
\text { angiotensina }\end{array}$ & 101 & $50,00 \%$ & 33 & $42,30 \%$ & 134 & $47,90 \%$ & 0,153 \\
\hline Alfa bloqueador adrenérgico & - & - & 1 & $1,30 \%$ & 1 & $0,40 \%$ & 0,135 \\
\hline $\mathrm{IECA}^{\star *}$ & 25 & $12,40 \%$ & 19 & $24,40 \%$ & 44 & $15,70 \%$ & 0,011 \\
\hline Vasodilatadores diretos & 6 & $3,00 \%$ & 5 & $6,40 \%$ & 11 & $3,90 \%$ & 0,167 \\
\hline Alfa agonistas de ação central & . & & 6 & $7,70 \%$ & 6 & $2,10 \%$ & 0 \\
\hline
\end{tabular}

Legenda: *AT1: Angiotensina 1; **IECA: Inibidores da enzima conversora de angiotensina

Fonte: Rezende VF, et al., 2021.

Sobre o controle da PA, observado na Tabela 3, averiguando-se, juntamente, as médias de PAS e de $\mathrm{PAD}$, houve descontrole pressórico em ambos os grupos, sendo o predomínio nos grupos de hipertensos com alteração da TFGe, com exceção das mulheres de etnia não branca.

Tabela 3 - Média de Pressão Arterial Sistólica (PAS) e Diastólica (PAD) associada a taxa de filtração glomerular estimada em hipertensos.

\begin{tabular}{|c|c|c|c|c|c|}
\hline ETNIA & n (\%) & $\begin{array}{c}\text { PAS } \\
\text { (média } \pm D P \text { ) }\end{array}$ & P-valor & $\begin{array}{c}\text { PAD } \\
\text { (média } \pm D P \text { ) }\end{array}$ & $p$-valor \\
\hline \multicolumn{6}{|c|}{ Etnia branca } \\
\hline \multicolumn{6}{|l|}{ Mulheres } \\
\hline TFGe $<60$ & $9(3,21 \%)$ & $133 \pm 17$ & \multirow{2}{*}{0,5606} & $71 \pm 14$ & \multirow{2}{*}{0,049} \\
\hline $\mathrm{TFGe} \geq 60$ & $48(17,14 \%)$ & $128 \pm 18$ & & $76 \pm 10$ & \\
\hline \multicolumn{6}{|l|}{ Homens } \\
\hline TFGe $<60$ & $12(4,28 \%)$ & $134 \pm 28$ & \multirow{2}{*}{0,5156} & $80 \pm 24$ & \multirow{2}{*}{0,6026} \\
\hline $\mathrm{TFGe} \geq 60$ & $111(39,64 \%)$ & $126 \pm 16$ & & $76 \pm 11$ & \\
\hline \multicolumn{6}{|c|}{ Outras etnias } \\
\hline \multicolumn{6}{|l|}{ Mulheres } \\
\hline TFGe $<60$ & $1(0,35 \%)$ & 66 & \multirow[b]{2}{*}{-} & 45 & \multirow[b]{2}{*}{-} \\
\hline $\mathrm{TFGe} \geq 60$ & $30(10,71 \%)$ & $123 \pm 11$ & & $74 \pm 10$ & \\
\hline \multicolumn{6}{|l|}{ Homens } \\
\hline TFGe $<60$ & $3(1,07 \%)$ & $151 \pm 43$ & \multirow{2}{*}{0,3501} & $78 \pm 10$ & \multirow{2}{*}{0,8775} \\
\hline $\mathrm{TFGe} \geq 60$ & $66(23,57 \%)$ & $130 \pm 21$ & & $77 \pm 14$ & \\
\hline
\end{tabular}

Fonte: Rezende VF, et al., 2021.

\section{DISCUSSÃO}

Os resultados do presente estudo reiteram a íntima relação evidenciada na literatura da hipertensão como causa e consequência de lesões renais (ZHANG L, et al., 2012). Foi encontrado uma alta prevalência de hipertensos com alteração da TFGe (27,86\%), vista também no estudo de Castro TLB, et al. (2020) e que tem importância no que tange a abordagem precoce desses pacientes com intuito de reduzir a progressão da 
injuria renal transitória em DRC. O padrão do paciente hipertenso com maior risco de desenvolver DRC foi caracterizado como: homem, branco, com 60 anos ou mais de idade, perfil também encontrado por outros autores (AGUIAR LK, et al., 2020; REZENDE NETA DS, et al., 2012).

Quanto às variáveis sociodemográficas, o sexo masculino esteve presente em $68,60 \%$ da amostra, havendo predomínio dentro do grupo de hipertensos com alteração da TFGe. Isso se justifica pelo fato de o sexo feminino possuir maior proteção aos danos renais relacionados à idade devido a diferenças ligadas ao estrógeno, que por sua vez, é responsável por conservar o óxido nítrico, molécula essencial na regulação do tônus vascular (COBO G, et al., 2016).

Em relação a etnia, a amostra obteve predomínio de brancos em relação à não brancos, sendo isto visto também dentro do grupo de hipertensos com alteração da TFGe, o que corrobora com dados de outros trabalhos, nos quais esta exerce papel influenciador na TFGe, em que os brancos, quando comparados às outras raças, possuem mais diagnóstico de alteração da função renal (SCHAEFER JCF, et al., 2015). De acordo com Peralta CA, et al. (2011), as diferenças encontradas entre as etnias podem ser explicadas por fatores genéticos, sociodemográficos e psicossociais, devido a maior exposição de certas populações a qualidade de vida desfavorável. No que se refere a idade, houve um predomínio de idade maior ou igual a 60 anos em $67,5 \%$ da amostra, fato observado de forma mais marcante dentro do grupo com alteração renal, e que se justifica pela redução fisiológica da quantidade de néfrons à medida que a idade avança, causando assim, a redução da TFGe, vista também em outro estudo (CASTRO TLB, et al., 2020).

No que se refere às comorbidades associadas, evidenciou-se mais casos de DM nos hipertensos com alteração da TFGe, fato também observado nos estudos de Aguiar LK, et al. (2020) e Pinho NA, et al. (2015). Essa relação entre a alteração da TFGe e DM pode ser explicada pela hiperfiltração e hiperperfusão renal, bem como a proteinúria e o estresse oxidativo pelo qual a hiperglicemia é responsável (PINHO NA, et al., 2015). Já em relação à dislipidemia, não houve diferença entre os grupos. No entanto, um estudo realizado no Oeste do Paraná com 136 pacientes renais crônicos demostrou uma prevalência de $75,7 \%$ de dislipidemia entre os participantes, sendo esse dado explicado pelos danos que a dislipidemia causa às células mesangiais e endoteliais dos rins (PERES LAB e BETTIN TE, 2015).

Acerca dos distúrbios de sódio e potássio, foi possível verificar uma maior prevalência de hipertensos com esses distúrbios em associação à alteração da TFGe do que o distúrbio isoladamente, sem alteração funcional dos rins. Isso reafirma a importância desses órgãos sobre o equilíbrio hidroeletrolítico, uma vez que a alteração da função renal, desregula o mecanismo de controle da PA realizado através da eliminação de sódio e água pela urina, levando à retenção destes íons (SANTOS TMP e VASCONCELOS SML, 2012). O mesmo ocorre com o potássio, que apesar de levar à redução dos níveis pressóricos através do estímulo à eliminação de sódio, pelo fato de a função renal estar comprometida, não exercerá seu papel, como também acumulará (BARROSO WKS, et al., 2020).

No que concerne aos hábitos de vida, o tabagismo possuiu menor predomínio nos hipertensos com alteração da TFGe, contrapondo-se ao estudo de Rezende Neta DS, et al. (2012), que relaciona o tabagismo a uma maior alteração da TFGe, o que se explica pela presença de gases e partículas nefrotóxicas que são inaladas ao fumar (ELIHIMAS JÚNIOR UF, et al., 2014). Por outro lado, foi possível relacionar o hábito de fumar com a presença da HAS, observado também nos estudos de Aguiar LK, et al. (2020), Pinho NA, et al. (2015) e Schaefer JCF, et al. (2015) e que pode ser explicado pelas alterações hemodinâmicas, como a elevação da frequência cardíaca e da PA, causadas pela nicotina quando esta se liga à certos receptores colinérgicos (ELIHIMAS JÚNIOR UF, et al., 2014).

Dos pacientes com alteração da TFGe, a maioria já estava em terapia dupla ou tripla, e a minoria em monoterapia com anti-hipertensivos. Os pacientes sem alteração também se encontravam, em sua maioria, em terapia dupla ou tripla. No entanto, a minoria desse grupo estava em tratamento com mais de três drogas, o que aponta a dificuldade de controle da PA, principalmente no que diz respeito à presença de alteração da TFGe (PINHO NA, et al., 2015; CASTRO TLB, et al., 2020).

É importante destacar também, o alto percentual de hipertensos com alteração da TFGe sem uso de qualquer medicação anti-hipertensiva no presente estudo. Isso reflete a grande dificuldade de adesão ao tratamento da HAS, que pode ter contribuído para a internação desses pacientes (PINHO NA, et al., 2015). 
Em ambos os grupos, os medicamentos mais usados foram os Bloqueadores do Receptor da Angiotensina (BRA), solidificando a escolha dos fármacos que atuam sobre o sistema renina-angiotensina-aldosterona como primeira linha no tratamento da hipertensão em pacientes com alterações renais, assim como os Inibidores da Enzima Conversora de Angiotensina (IECA), classe mais utilizada em um estudo realizado em São Paulo (PINHO NA, et al., 2015). Entretanto, a classe dos IECA, no presente estudo, apresentou baixo uso, o que pode ser elucidado pelo seu efeito colateral mais comum, que é a tosse seca resultante do acúmulo de bradicinina nos brônquios ao inibir a enzima conversora de angiotensina. Por diminuírem consideravelmente a proteinúria e microalbuminúria essas duas classes de anti-hipertensivos são de grande valia no tratamento dos pacientes com HAS e DRC (BARROSO WKS, et al., 2021; MENNUNI S, et al., 2013; BENIGNI A, et al., 2011).

Outros medicamentos de destaque, usados em associação aos IECA e BRA, são os Beta Bloqueadores e os Diuréticos de Alça. Os Beta Bloqueadores, segunda classe medicamentosa mais utilizada pelo grupo com alteração da TFGe, são indicados aos pacientes que, além de hipertensos com repercussão renal, tenham doença arterial coronariana e/ou insuficiência cardíaca associada, pois além de causar redução da secreção de renina e catecolaminas, também reduz o débito cardíaco (BARROSO WKS, et al., 2021). Já os diuréticos de alça, terceira classe medicamentosa de maior uso no presente estudo e no trabalho de PINHO NA, et al., (2015), são empregados em casos de DRC em estágios mais avançados, atuando sobre a retenção de sódio e água.

Sobre o controle da PA, correlacionando os níveis pressóricos médios diastólicos e sistólicos, a maioria dos pacientes estava com a PA alterada, sendo que os hipertensos com alteração da TFGe obtiveram maior descontrole, com exceção das mulheres de etnia não branca, que não obtiveram quantidade mínima de participantes necessária para a realização dos testes estatísticos. Dessa forma, reafirma-se, assim como nos estudos de Sarafidis PA, et al. (2017), Mennuni S, et al. (2013) e Silva RLM (2017), a intensa relação entre o controle da PA e a integridade funcional dos rins, e a importância do rastreio da redução da TFGe em hipertensos, como forma de prevenir o retardo das diversas injúrias renais para DRC.

Apesar de o estudo apresentar informações de impacto sobre a relação da HAS e alteração da TFGe, é preciso registrar suas limitações. Por ser um estudo retrospectivo transversal, não foi possível estabelecer relação de causa e efeito, pois não houve um grupo controle (ensaio clínico) para testar o que de fato é um fator protetor ou de risco e a resposta às medicações comparando com um grupo placebo. Os prontuários foram preenchidos por vários médicos, ou seja, não houve padronização dos mesmos. A causa da internação e o estado hemodinâmico dos pacientes não foram averiguados, não sendo possível atribuir a alteração da PA exclusivamente à HAS e alteração da TFGe. Não foi possível distinguir os pacientes com alterações transitórias da TFGe daqueles com alterações crônicas. A aferição da PA foi realizada por vários profissionais e com diferentes equipamentos, não havendo certeza da calibração prévia dos esfigmomanômetros utilizados.

\section{CONCLUSÃO}

A prevalência de alteração da TFGe em hipertensos foi alta, tendo sido encontrado como fator associado o DM e os distúrbios hidroeletrolíticos. Hipertensos com alteração da TFGe tem maior dificuldade em controlar a PA, estando a maioria em uso de duas ou mais classes medicamentosas anti-hipertensivas. A identificação dos hipertensos com alteração da TFGe possibilita a implementação de medidas preventivas que possam modificar a progressão das injúrias renais para DRC e/ou da própria HAS para estágios mais avançados.

\section{AGRADECIMENTOS}

Aos Professores Guillermo Patricio Ortega Jácome, Danielle Cristina Zimmermann Franco, Ricardo Fernandes Rezende, Maria Augusta de Mendonça Lima e Cássio dos Santos Nunes pelo empenho dedicado à elaboração deste trabalho, pelas suas correções e incentivo. Á Instituição de Ensino Centro Universitário Presidente Antônio Carlos (UNIPAC) de Juiz de Fora - MG e ao Hospital Dr. João Felício de Juiz de Fora MG pela oportunidade e fornecimento de dados que foram fundamentais para a realização deste trabalho. 


\section{REFERÊNCIAS}

1. AGUIAR LK, et al. Fatores associados à doença renal crônica: inquérito epidemiológico da Pesquisa Nacional de Saúde. Revista Brasileira de Epidemiologia, 2020; 23: E200044.

2. AOKI BB, et al. Lesão renal aguda após exame contrastado em idosos. Rev. Latino-Am. Enfermagem, 2014; 22(4): 637-44.

3. BARRETO MS, et al. Conhecimento sobre a hipertensão arterial e fatores associados à não adesão à farmacoterapia. Rev. Latino-Am. Enfermagem, 2014; 22(3): 484-490.

4. BARROSO WKS, et al. Diretrizes Brasileiras de Hipertensão Arterial - 2020. Arquivos Brasileiros de Cardiologia, 2021; 116(3): 516-658.

5. BENIGNI A, et al. Inhibiting angiotensin-converting enzyme promotes renal repair by limiting progenitor cell proliferation and restoring the glomerular architecture. The American Journal of Pathology, 2011; 179(2): 628-638.

6. BUCHARLES SGE, et al. Hypertension in patients on dialysis: diagnosis, mechanisms, and management. Brazilian Journal of Nephrology, 2019; 41(3): 400-411.

7. CARVALHO MV, et al. A influência da Hipertensão Arterial na Qualidade de Vida. Arq Bras Cardiol, 2013; 100(2): 164174.

8. CASTRO TLB, et al. Função renal alterada: prevalência e fatores associados em pacientes de risco. Rev Cuid, 2020; 11(2): e1019.

9. CERQUEIRA DP, et al. Fatores preditivos da insuficiência renal e algoritmo de controle e tratamento .Rev. Latino-Am. Enfermagem, 2014; 22(2): 211-7.

10. COBO G, et al. Sex and gender differences in chronic kidney disease: progression to end-stage renal disease and haemodialysis. Clinical Science, 2016; 130: 1147-1163.

11. ELIHIMAS JÚNIOR UF, et al. Tabagismo como fator de risco para a doença renal crônica: revisão sistemática. J Bras Nefrol, 2014; 36(4): 519-528.

12. FALCÃO AS, et al. Estilo de Vida e Adesão ao Tratamento de Hipertensão Arterial Sistêmica em Homens Idosos. Revista Brasileira de Promoção a Saúde, 2018; 31(2): 1-10.

13. FIGUEIREDO NN, ASSAKURA L. Adesão ao tratamento anti-hipertensivo: dificuldades relatas por indivíduos hipertensos. Acta Paulista de Enfermagem, 2010; 23(6): 782-787.

14. GOUVEIA MMA, et al. Gênese e Fatores de Risco para a Hipertensão Arterial Genesis and Risk Factors for Hypertension. Rev. Bras. Hipertens, 2018; 25(1): 6-12.

15. MENDONÇA LBA, et al. Acidente Vascular Encefálico como complicação da Hipertensão Arterial: Quais são os fatores intervenientes? Escola Anna Nery, 2012; 16(2): 340-346.

16. MENNUNI S, et al. Hypertension and kidneys: unraveling complex molecular mechanisms underlying hypertensive renal damage. Journal of Human Hypertension, 2013; 28: 74-79.

17. MORAES CE, et al. Preditores de insuficiência renal crônica em pacientes de centro de referência em hipertensão arterial. Revista da Associação Médica Brasileira, 2009; 55(3): 257-262.

18. MARTINS NETO UR, et al. Prevenção da doença renal crônica à luz da perspectiva de pessoas hipertensas. Revista Eletrônica Acervo Saúde, 2021. 13(5): 1-9.

19. OLIVEIRA BL, et al. A influência da Estratégia Saúde da Família no uso de serviços de saúde por adultos hipertensos no Brasil. Rev. Bras. Epidemio, 2020; 23: 1-14.

20. OLIVEIRA FJS, et al. Atuação do enfermeiro na prevenção de doença renal crônica em portadores de diabetes: revisão integrativa. Revista Eletrônica Acervo Saúde, 2019; 30: 1-5.

21. OLIVEIRA JL, et al. O combate a hipertensão arterial na estratégia e saúde da família: uma revisão bibliográfica. Revista Eletrônica Acervo Saúde, 2021; 13(2): 1-7.

22. PAN AMERICAN HEALTH ORGANIZATION. World Hypertension Day 2020. 2020. Disponível em: https://www.paho.org/pt/campanhas/dia-mundial-da-hipertensao-2020. Acessado em: 10 de julho de 2021.

23. PERALTA CA, et al. Racial and ethnic differences in kidney function decline among persons without chronic kidney disease. Journal of the American Society of Nephrology, 2011; 22(7): 1327-1334.

24. PERES LAB, BETTIN TE. Dislipidemia em pacientes com doença renal crônica. Rev Soc Bras Clin Med, 2015; 13(1): 10-3.

25. PINHO NA, et al. Hypertensive patients with and without kidney disease: assessment of risk factors. Revista da Escola de Enfermagem da USP, 2015; 49: 101-108.

26. REZENDE NETA DS, et al. Avaliação renal de hipertensos pela clearance de creatinina num centro de saúde de Teresina-PI, Brasil. Revista de Enfermagem Referência III, 2012; 3(6): 25-31.

27. SANTOS TMP, VASCONCELOS SML. Ingestão de Na+ e K+ versus HAS: bases para o seu manejo e protocolo de investigação. Revista Brasileira de Hipertensão, 2012; 19(2): 51-55.

28. SARAFIDIS PA, et al. Hypertension in dialysis patients: a consensus document by the European Renal and Cardiovascular Medicine (EURECA-m) working group of the European Renal Association-European Dialysis and Transplant Association (ERA-EDTA) and the Hypertension and the Kidney working group of the European Society of Hypertension (ESH). Nephrology Dialysis Transplantation, 2017; 32(4): 620-640.

29. SCHAEFER JCF, et al. Estimativa da função renal na população de 18 a 59 anos da cidade de Tubarão-SC: Um estudo de base populacional. Jornal Brasileiro de Nefrologia, 2015; 37(2): 185-191.

30. SILVA RLM. Hipertensão no doente renal crônico: antes e depois do transplante renal. Dissertação (Mestrado em Medicina) - Instituto de Ciências Biomédicas de Abel Salazar. Universidade do Porto, Porto, Portugal, $2017 ; 31$ p.

31. SOUZA SR, et al. Doença renal crônica: Informações de enfermagem aos envolvidos acerca da gravidade do consumo da carambola. Revista Eletrônica Acervo Saúde, 2020; 12(12): 1-7.

32. ZHANG L, et al. Prevalence of chronic kidney disease in China: a cross-sectional survey. The Lancet, 2012; 379(9818): 815-822. 